\title{
Rites De Reintegration "Afolile" Des Femmes Adulteres Chez Les « Fon » D'abomey : Elements Pour Une Socio- Anthropologie De La Purification
}

\author{
Charles Lambert Babadjide \\ Enseignant au Département de Sociologie-Anthropologie \\ de l'Université d'Abomey-Calavi (UAC), Bénin
}

Doi:10.19044/esj.2021.v17n7p170

Submitted: 20 November 2020

Accepted: 12 January 2021

Published: 28 February 2021
Copyright 2021 Author(s)

Under Creative Commons BY-NC-ND

4.0 OPEN ACCESS

Cite As:

Babadjide C.L. (2021). Rites De Reintegration "Afolile" Des Femmes Adulteres Chez Les « Fon » D'abomey : Elements Pour Une Socio-Anthropologie De La Purification. European Scientific Journal, ESJ, 17(7), 170. https://doi.org/10.19044/esj.2021.v17n7p170

\section{Résumé}

La présente production met en évidence l'analyse des mobiles autour de l'adultère ainsi que les méthodes mises en place par les groupes socioculturels « fon » d'Abomey pour réintégrer la femme adultère. L'étude est de nature qualitative. La collecte des informations est faite auprès de 92 personnes grâce aux techniques de collecte telles que la recherche documentaire, l'entretien individuel et l'observation directe. L'échantillon de recherche a été défini grâce aux techniques non probabilistes. Le traitement des données est fait manuellement puis elles sont soumises à l'éclairage de la théorie fonctionnaliste. Les résultats rendent compte d'une multiplicité d'acteurs dont l'intervention répond à des logiques parfois contradictoires du fait des vécus dans le milieu. Les données produites et analysées renseignent sur le fait que les rites de purification (afolile) sont des stratégies développées par la culture « fon » d'Abomey pour maintenir l'équilibre du couple et la cohésion familiale.

Mots clés : Fon d'Abomey, Réintégration Social, Adultère, Rites, Cohésion Familiale 


\title{
Rites De Reintegration "Afolile" Des Femmes Adulteres Chez Les « Fon » D’abomey : Elements Pour Une Socio- Anthropologie De La Purification
}

\author{
Charles Lambert Babadjide \\ Enseignant au Département de Sociologie-Anthropologie \\ de l'Université d'Abomey-Calavi (UAC)/Bénin
}

\begin{abstract}
This production highlights the analysis of the motives around adultery and the methods put in place by Abomey's "fon" socio-cultural groups to reintegrate adultery women. The study is qualitative in nature. Information is collected from 92 people through collection techniques such as documentary research, personal interview and direct observation. The research sample was defined using non-probabilistic techniques. Data processing is done manually and then they are exposed to functionalist theory. The results reflect a multiplicity of actors whose intervention responds to sometimes contradictory logics due to the experiences in the environment. The data produced and analyzed indicate that purification rites (afolile) are strategies.
\end{abstract}

Keywords: Abomey's Foundation, Social Reintegration, Adultery, Rites, Family Cohesion

\section{Introduction}

La recomposition des systèmes de valeurs et de contre valeurs renseigne sur l'évidence de nouvelles orientations, de nouveaux enjeux et de nouvelles références ou tout simplement sur les formes expressives d'une société en "reconstruction". Or, construire la vie conjugale comme une réalité sociale dynamique, c'est admettre que le lien conjugal, son maintien et ses instabilités ne répondent nécessairement plus aux mêmes lois que dans le passé. C'est fort de cet état de chose que F. De Singly (1996, p. 89), soutient que la relation élective conjugale a supplantée tous les autres liens de parenté et est devenue le lieu d'une quête d'émancipation personnelle. C'est dire que la problématique de l'émancipation est aussi au cour de la vie conjugale. De ce fait, l'homme de par sa nature, est fait pour vivre en communauté. Il est un être social car, c'est au contact de ses semblables qu'il peut acquérir les règles de « civilité » qui lui permettent de vivre en paix avec autrui. La vie en société désigne une intégration de chaque individu dans le milieu humain qui le structure et lui donne sens. Tout ce que l'homme fait, est toujours et déjà au sein de la société. 
Ainsi, les hommes se regroupent tout d'abord en famille (premier lieu des relations sociales) puis en village et enfin en cité, ceux-ci n'étant rien d'autres que la communauté. Cette conception de la cellule sociale de base qu'est la famille et les valeurs fondamentales qui la sous-tendent traduisent un idéal de famille dont la pérennité constitue une préoccupation majeure (à l'échelle macro) comme l'exprime un dicton fon selon lequel "même si le monde s'écroule, il n'entraîne pas dans sa chute le «"hønnu" c'est-à-dire la famille ». Ce dicton traduit l'attachement de la société «fon » d'Abomey à l'unité, à la consolidation des liens sociaux et à la stabilité durable du ménage en dépit de l'éclatement des repères qui jalonne le XXI ${ }^{\text {ème }}$ siècle. Or, l'observateur le moins averti constate aujourd'hui une déstabilisation progressive de la dynamique familiale et communautaire béninoise frappée par toutes sortes de dissociations et de déviances. En effet, les mutations sociales, qu'a connu le Dahomey au lendemain de son indépendance, ont bouleversé les modes de vie et changé les rapports sociaux. Cette évolution n'a pas épargné la structure de la famille. Elle a produit un effet en agissant sur l'un de ses fondements les plus profonds : le mariage. Si le mariage (suivant les règles coutumières ou non) était perçu comme une garantie de l'équilibre de la vie sociale par la sécurité, la stabilité et la chaleur humaine (D. Paquett, 2004, p. 213), ce n'est plus systématiquement le cas de nos jours. L'observation empirique des vécus dans les ménages d'Abomey permet de remettre en cause l'idée d'une stabilité durable des alliances conjugales à cause surtout de l'adultère.

L'adultère au sein des couples entraine fréquemment des séparations de nombreux conjoints. Longtemps interdit, exceptionnel, marginal, signe d'une instabilité familiale, d'une crise de l'individu et de la société, l'adultère est devenu aujourd'hui un acte courant et banalisé, un choix individuel. Il constitue l'un des problèmes qui fragilise les couples en général et en particulier des couples en milieu «fon»d'Abomey. Il génère de grands préjudices à la stabilité des couples et à l'unité de la famille. Bien qu'il ne s'agisse pas d'un phénomène social nouveau, sa place dans les consciences individuelles et collectives a pris une autre dimension au cours des dernières années. L'adultère et l'infidélité ont donc pris une proportion inquiétante dans nos sociétés. Les considérations coutumières, pourtant encore d'actualité dans les collectivités d'Abomey et l'emprise de l'expérience religieuse dans les ménages n'empêchent pas l'évidence de fractures sociales, de clivages et de ruptures entre les conjoints qui ont vécu ce phénomène. Si pendant longtemps l'infidélité était considéré comme l'apanage des hommes (G. Flaubert, 2006, p. 145), on remarque, qu'elle a gagné du terrain du côté de la gente féminine. Il n'est plus un secret de polichinelle que bon nombre de femmes mariées mènent de nos jours une double vie. Cette violation de la fidélité conjugale de la femme, est une faute qui demeure le point de mire des organisations de 
droits de l'homme, des religieux et sociologues, etc. Dans le monde, peu de sociétés criminalisent l'adultère de l'homme. En revanche l'adultère de la femme a toujours fait l'objet de condamnation. Ainsi, l'infidélité des femmes a longtemps été sanctionnée par la peine de mort (G. Leleu, 2004, 125). Dans le christianisme par exemple, l'adultère est condamné tout aussi formellement dans le Nouveau Testament.

En Afrique, chaque société à sa propre manière de corriger les déviances. Ainsi, l'adultère est diversement corrigé par la tradition à travers les rites. Le rite, vu comme l'ensemble des «comportements codifiés et imposés par le groupe social » (J. Cazeneuve, 1971, p. 106), est inhérent à la vie de toute société humaine. Il pénètre la totalité des activités humaines ; la culture, la quotidienneté, tout autant que les grands moments de la vie villageoise (L.V. Thomas et R. Lumeau, 1986, p. 178). Dans la panoplie de rites existants, le rite de purification est celui qui, aujourd'hui, intervient le plus dans les cérémonies de réparation d'une faute grave dans la communauté traditionnelle. En effet, au Bénin, chaque société à ses propres méthodes de purification pour la correction et la réintégration de la femme adultère. Les cérémonies de purification donnant droit à la femme adultère de regagner son foyer. Cette pratique varie d'une ethnie à une autre et n'est donc pas propre à une société. Dans certaines ethnies, la femme adultère ne peut regagner le foyer conjugal qu'après avoir été purifiée au cours d'une cérémonie spéciale. Les "fon" d'Abomey font partie de ces ethnies du Bénin qui restent attachées à ces traditions et donnent une seconde chance à la femme adultère par le biais des rites de purification dits "Afjlile". Malgré que l'adultère soit corrigé par des rituels traditionnels qui passent par les rites de réintégration et malgré le caractère dissuasif de ce procédé, on constate une persistance de ce phénomène chez les femmes «fon » d'Abomey. Face à cette situation, une interrogation s'impose à nous : quel est le rôle du rituel "Afolile" à la femme adultère d'Abomey?

\section{Matériels et méthodes}

Portant sur l'analyse socio-anthropologique des rites de réintégration "afolile" des femmes adultères chez les «fon »d'Abomey, cette recherche est d'obédience qualitative. Ce volet qualitatif donne un aperçu des comportements et des perceptions des personnes ciblées par l'enquête. Cela a permis d'étudier leur opinion sur le sujet. La collecte des données empiriques, ne pouvant atteindre toute la population, un recours aux techniques d'échantillonnage de boule de neige et de choix raisonné ont été mises à profit pour définir l'échantillon de recherche. Elles ont permis de prendre en compte des unités de recherche constituées de femmes mariées, de femmes adultères, des tradi-praticiens, des chefs religieux, des tangninon, des hommes victimes d'adultère et de l'entourage immédiat des couples victimes. Soit cinquante- 
deux (52) personnes ont été sélectionnées. Ces acteurs sont identifiés, pour la plupart, par choix raisonné. Les critères de choix varient en fonction des catégories d'acteurs. Les principaux critères tiennent compte de l'expérience des chefs religieux par rapport à la résolution des conflits conjugaux. Aussi, à partir des échanges avec l'entourage immédiat des couples victimes d'adultère, il a été identifié quelques situations pouvant conduire à l'adultère. A chaque catégorie d'acteurs, est associé, un canevas de départ en termes de types d'informations attendues. Trois techniques de collecte de données ont été employées pour mener à bien la collecte des données empiriques. Il est question de la recherche documentaire, de l'entretien semi-structuré et de l'observation.

Les données collectées sont structurées de façon à ce qu'elles puissent être analysées efficacement. La première étape de notre travail consiste à la transcription intégrale des informations recueillies auprès de chaque personne interrogée dans un logiciel de traitement de texte (Word). Ensuite, a suivi le traitement des données. Pour ce faire, les informations recueillies par le biais des guides d'entretien, ont été soumises à un traitement qui a consisté à rechercher des similitudes dans les descriptions et à faire des catégorisations par axe d'analyse et à en faire une synthèse. La structuration des données qualitatives a paru exigeante en réflexion et a nécessité aussi assez de temps. Nous avons par la suite fait usage de l'analyse de contenu pour la catégorisation des informations issues du guide d'entretien. Ces analyses ont suivi les axes de la structuration faite lors du traitement; elle a consisté en un croisement comparatif des informations, soit à l'intérieur de la même catégorie d'acteurs, soit en inter-catégorie ; ensuite à faire une comparaison des éléments dégagés de l'analyse avec les tendances ressorties de la littérature.

Cité historique, la commune d'Abomey s'étend sur une superficie de $142 \mathrm{~km}^{2}$. Elle est limitée au Nord par la commune de Djidja, au Sud par la commune d'Agbangnizoun, à l'Est par la commune de Bohicon et à l'Ouest par la commune d'Aplahoué. Elle compte sept (07) arrondissements subdivisés en vingt-neuf (29) quartiers de ville et villages. 
Carte 1. Présentation de la commune d'Abomey

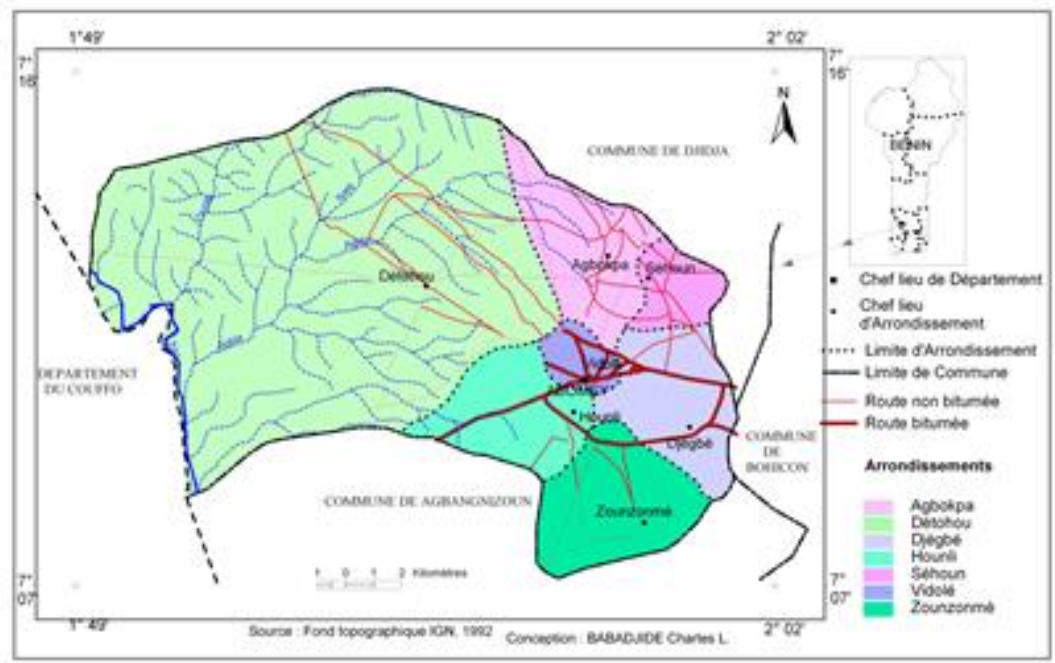

Source : Conception Babadjidé C. L., IGN, 1992.

La densité humaine de la commune d'Abomey est de 468 habitants $/ \mathrm{km}^{2}$. La population de la commune d'Abomey est composée majoritairement de Fon. Cette commune est une localité où les pratiques ancestrales demeurent encore prégnantes dans la mesure où l'influence des chefs religieux traditionnels est incontestable dans tous les arrondissements. Cependant, on y observe la cohabitation de plusieurs religions. L'ensemble de ces informations renseignent sur les dynamiques sociales influençant l'expérience de l'adultère dans la commune d'Abomey. Le choix d'Abomey constitue donc une grappe assez représentative pour une étude de l'adultère au Bénin.

\section{Résultats et discussion : Rites de purification de la femme adultère chez les « fon » d'Abomey}

Cette partie est consacrée à la présentation, l'analyse et la discussion des résultats de terrain. Les résultats montrent les mécanismes de purification de la femme adultère et des processus culturels de réinsertion des ces dernières.

\section{La gestion culturelle de l'adultère}

Malgré les disfonctionnements observés au sein du foyer chez les « fon » d'Abomey, ceux-ci s'attachent au bien-être du couple afin d'assurer sa stabilité. Pour que cette stabilité soit garantie, chaque groupe socio-culturel dispose des moyens ou stratégies relatifs au contrôle social afin de mieux gérer les déviances pour s'assurer du maintien de l'harmonie et de la cohésion sociale. Mais la fidélité au foyer est le principal centre d'intérêt identifié. A 
cet effet, l'adultère encore appelé rapports sexuels extraconjugaux fait l'objet d'une attention particulière. En dehors des dispositions prises sur le plan juridique et de l'information apportée par les religions importées (qui montrent d'ailleurs leurs limites dans la réduction de l'infidélité), la tradition africaine aussi propose des mesures palliatives à cette préoccupation. C'est alors pourquoi, un sage a raconté à cet effet :

"Ce sont les mannes de nos ancêtres qui s'occupent du sort des femmes infidèles. Par exemple à l'entrée même de certaines maisons, il y a le fétiche Minonnan implanté à l'entrée de la maison comme gardien. Quand une femme commet l'adultère, déjà à l'entrée de la maison, le fétiche se transforme en une bête féroce, chez d'autres, en grand arbre qui se déplace et l'empêche de rentrer dans la maison. Elle commence par crier comme une folle. Chose curieuse, il n'y a qu'elle seule qui les voit.»[Entretien avec Msr A. A., 74ans, marié et père de cinq enfants.]

A la suite des propos de cet enquêté, il a été compris que, la société indigène nous parait armée pour opérer son redressement. Le fétiche Minonnan est le fétiche protecteur des femmes de la maison comme l'indique son nom "Minən" notre mère, "Nan" reine c'est-à-dire la reine mère des femmes de la concession. Il est implanté à l'entrée de la maison comme ange gardien des femmes. Alors quand une femme de cette maison commet le crime d'adultère, à son arrivée automatiquement le fétiche sent qu'il y a un être impur qui veut pénétrer dans la maison, aussitôt, il se transformerait en des choses mystiques dont leur explication dépasse même la compétence scientifique. Comme le montre la photo ci-dessous, le fétiche Minonnan présente l'aspect d'un Tolègba. Mais en réalité ce n'est pas un Tolègba. Le Tolègba reste à l'entrée du village tandis que le fétiche Minonnan est destiné à la maison. En voici une illustration.

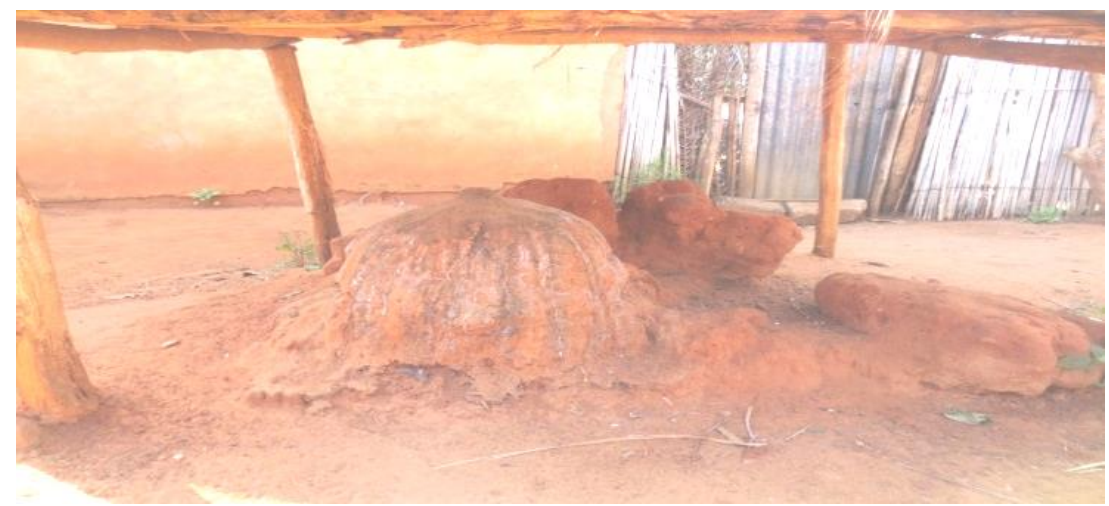

Photo 1. Le fétiche MINつNNAN, Abomey, Hounli.

Prise de vue : Babadjidé, 2019 
Il a été aussi révélé que, quand la femme commet l'adultère, garde le silence et continue à préparer pour son mari, les femmes défuntes de la maison ayant commise cet acte qui a été la source de leur mort se révoltent contre cette femme et souhaitent à tout prix qu'elle les rejoigne. N'est-ce pas une manière pour décourager l'adultère dans ce milieu ? Mais ces déclarations ne sont pas fondées, surtout sur le plan scientifique. Car ce ne sont que des choses imaginaires, sans fondement, sans preuve, sinon comment se fait-il que les femmes défuntes se révoltent or nul ne sais ce qui se passe après la mort. Il faut alors une complémentarité entre la science et la tradition afin de mieux cerner le mystique.

\section{Le "fâ" comme guide dans le contexte d'adultère}

Dans la tradition «fon », consulter, prendre, fouiller, ou encore étaler le "fâ", est une démarche habituelle. Lorsqu'on constate dans un foyer, après des années de vie commune sans difficulté, la chute des affaires de l'époux, la faiblesse de son organisme face à certaines maladies qui autrefois était bien résistant, on commence par se poser des questions. Alors les soupçons de trahison conjugale (adultère) de la femme commencent. Le mari commence par se poser des questions sur la fidélité de sa femme. Si la femme ne fait pas l'aveu en cas d'infidélité, et continue de préparer pour le mari, c'est une malédiction pour le foyer. Soit elle a problème de fécondité, soit les enfants tombent malade ou ils meurent. Quand le mari est au courant et par honte, il ne dit rien à la famille, il tombe malade et souvent ce sont des maladies qui échappent à la médecine moderne. En ce moment le "fâ" intervient comme un éclaireur.

"Le "fâ" dans le panthéon vodoun est une divination très chère au peuple "fon ", il est l'Alpha et l'Omega de toutes ses cérémonies traditionnelles. Il est le messager entre Dieu et les hommes. Il est un guide spirituel, un éclaireur qui permet d'avoir une vision claire par rapport à une situation. Le "fâ" est également un pouvoir divinatoire destiné à révéler aux victimes la faute commise». [Entretien avec Dah TOKANME, Bokonon 65, marié et père de vingt-deux enfants]

En d'autres termes, c'est à travers les révélations du "fâ", que les populations d'Abomey arrivent à comprendre ce qui se passe dans le monde invisible. De là, on découvre le crime commis, au cas où la femme suspectée nie les faits. La plupart des cas, c'est quand les signes commencent par apparaitre qu'on consulte le "fâ". Celui-ci révèle que la femme garde un secret qu'elle devra révéler pour sa survie, celle de son époux ou celle de ses enfants. A partir de cet instant, le soupçon est établi et la famille est alertée pour amener 
la coupable à faire ses révélations. Les aveux une fois faits, un jour est retenus pour la cérémonie de purification.

\section{Les différentes étapes de la cérémonie de purification}

Dans le but de mieux comprendre la réaction qui suit ce péché en milieu «fon », et les éventuelles démarches à mener pour ne pas subir la colère des divinités traditionnelles, nous nous sommes rapprochés d'un chef de collectivité, une personne ressource issue de cette ethnie. Il nous explique ici les différentes étapes nécessaires à franchir avant de faire purifier la femme adultère. Selon lui, les étapes sont multiples. Si la femme avoue son adultère à son époux, il se refuse de le garder seul par devers lui et en informe l'ainé de sa concession. Il l'informe des soupçons qu'il a de sa femme et confirme l'acte d'adultère qu'elle a commis. Ce dernier fait recours au chef de la collectivité (hennuto). Le hennuto après l'avoir longuement écouté et mesurant la portée de la situation, réunit toute sa cour et invite la femme à faire les révélations publiquement, c'est-à-dire devant la cour. La femme prêtresse de la maison dite (Tangninon), après discussion avec le mari lui demanda sa décision face à la situation. Si il aime encore sa femme et veux toujours la garder, il va demander la grâce du pardon des ancêtres pour son épouse ceci marqué par la réalisation du rituel de purification de la femme adultère "AfJlile" dans sa famille afin de toujours la garder et aussi de maintenir la paix au sein de son foyer. On comprend donc que le rituel est en quelque sort un canal par lequel l'homme communique avec les Dieu afin de leur demander une doléance. Donc si l'homme décide de garder sa femme, ils doivent nécessairement passer par ce canal qui constitue un moyen pour le maintien de l'ordre social dans nos sociétés traditionnelles.

Quelques temps après les interrogations par rapport à la décision de l'homme, la Tangninon va lui faire la liste des différents ingrédients nécessaires pour la cérémonie. Notons que c'est la femme seule qui a le devoir de payer les ingrédients avec ses propres moyens. L'homme ne mettra pas un centime concernant quelconque dépense pour la cérémonie. Au pire des cas, l'époux jette de l'argent par terre et la femme ramasse. Il n'a pas le droit de lui remettre ça à main propre. Après cette étape, le jour de la cérémonie proprement dite est fixé.

\section{La cérémonie "Afokloklo" ou "Afłlile" proprement dite}

Le jour de la cérémonie, le hennuto convoque une séance familiale extraordinaire où la Tangninon, les femmes de la maison du mari dite (houessi), ses frères et sœurs ainé sont convoqués ainsi que les parents de la femme. Après l'installation des conviés, le hennuto désigne la Tangninon, à accorder la parole à la femme infidèle. Celle-ci passe à l'aveu en public exposant les faits ou les raisons l'ayant poussé à l'acte, elle a aussi l'obligation 
de citer le nombre d'hommes avec qui elle a couché "Assouhiha", tout ceci accompagné de promesses de ne plus recommencer. Suite à cela des conseils, colères, et réprimandes viennent sur son comportement mais aussi sur celui du mari. Après cela une longue interrogation de la Tangninon au mari débuta, pour s'assurer de faire tous les rituels qu'il faut dans le cas où il aurait couché sciemment ou inconsciemment avec sa femme après son forfait. Dans les deux cas où la réponse s'avère positive, le mari est attaché et on le frappe avec les rameaux (Azan) devant la famille. Après cette étape, la femme coupable est

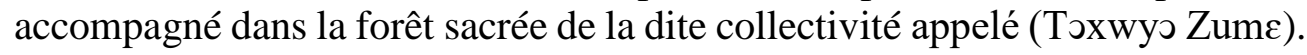

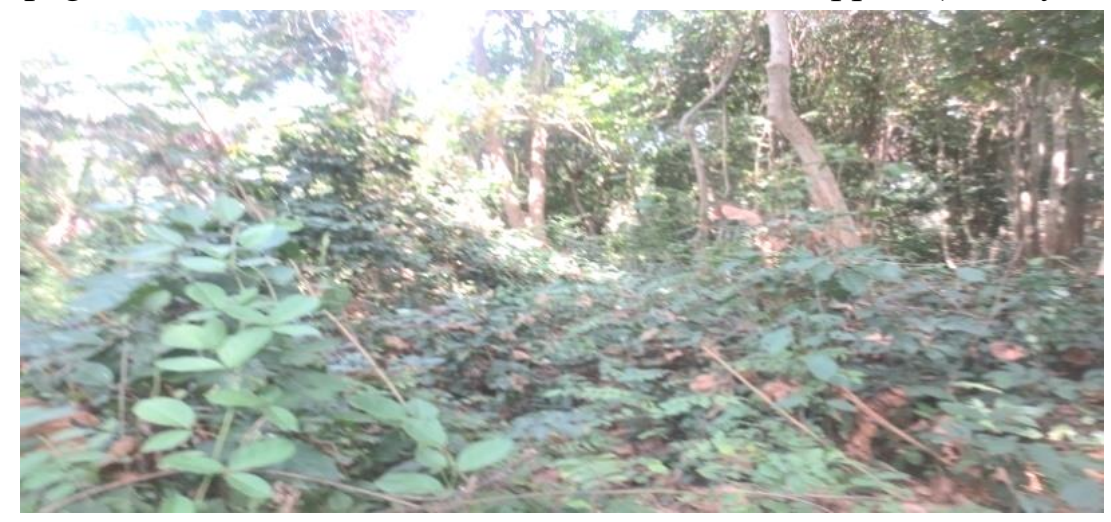

Photo 2. La forêt du TDXWYD dit TOXWYD ZUME

Prise de vue : Babadjidé, 2019.

La photo ci-dessus présente des arbres et arbustes. Il s'agit d'une forêt sacrée. C'est-à-dire le lieu où se font les cérémonies d'ordre traditionnel. Dans le cadre de cette étude, il s'agit du lieu où se font les cérémonies de purification de la femme adultère. A la sortir de cette forêt après la cérémonie, la femme devient pur, sans péché aux yeux des ancêtres. On peut donc conclure que c'est le lieu de rencontre entre la femme adultère et l'ancêtre de la collectivité (T⿰xwyว). Une fois dans la forêt, elle remet au Toxwyonon (le représentant du toxwy dans le monde des vivants) tous les objets dont elle s'est parée le jour de l'acte, à savoir : les perles, l'ensemble vestimentaire, ses bracelets, ses boucles d'oreilles, son slip etc. Après que le Toxwyวnon aie prit les objets, le Hounsso (celui qui est chargé de l'immolation), passe à l'immolation des animaux. Le sang de ces animaux est versé par terre devant le Toxwyว de la famille pour implorer sa clémence. 


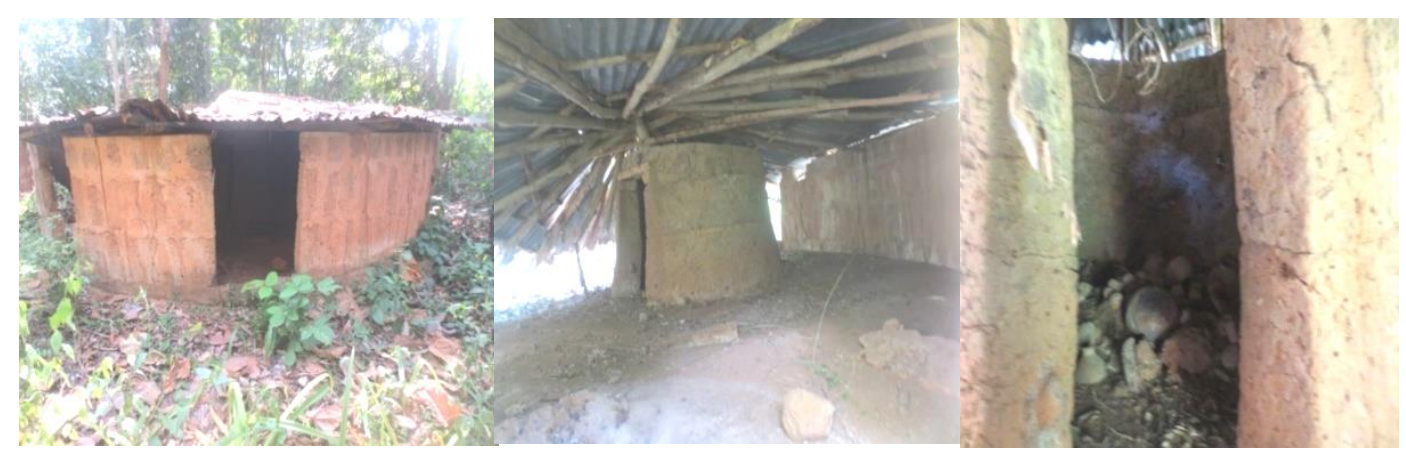

Photo a. Grande case Toxwy Photo b. Petite case dans la grandn Photo c. Petites jarres Planche 1 : La case du Toxwyo dit Toxwo Ho et le Toxwyo à Abomey Vidolé.

Prise de vue : Babadjidé, 2019

La planche ci-dessus présent une case et à l'intérieur de celle-ci se trouve une autre qui contient de petites jarres de forme différentes, des calebasses... il a été révélé sur le terrain que ces objets représentent le Təxwyว. On peut donc conclure que l'esprit du Toxwyว se cache à l'intérieur de cette case et en faisant la cérémonie devant celle-ci signifie que c'est en présence du Toxwy que la cérémonie se fait. A la suite de l'immolation, le Toxwyonon cherche des feuilles sacrées dans la forêt (Ayaman, déssréssigèman, ahissihissi etc...) et y ajoute quelques feuilles appelées (Aman dokpo) les triture pour en faire une tisane.

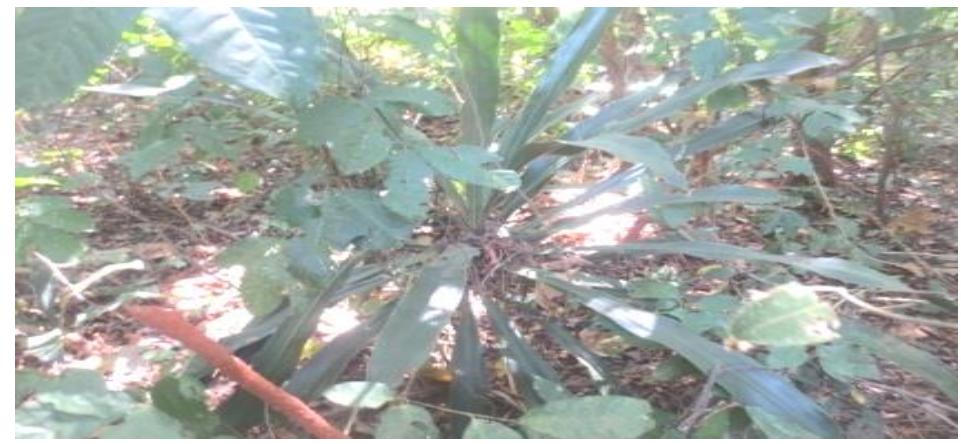

Photo 3. Feuille Ayaman, à Abomey Hounli.

Prise de vue : Babadjidé, 2019

La photo 3 présente des arbustes et au milieu de ces arbustes se trouve la plante de la feuille d'Ayaman qui est l'une des feuilles utilisées au cours de la cérémonie. En effet, il se présente comme une fleur à plusieurs branches longues dont chaque branche constitue la feuille "Ayaman". Qui est aussi utilisée lors de la cérémonie. Après la trituration, le Toxwyonon divise la tisane en deux et les met dans deux jarres : il met de l'eau chaude dans une jarre et de l'eau froide dans l'autre jarre afin que la femme en fasse sa toilette intime. Suite à cela, la Toxwyossi ou la dénin (celle qui suit directement le 
Toxwyวnon) ou en quelque sorte (le Toxwyənon féminin) aide la femme à se laver et on passe à l'étape de rasage des cheveux. Le Toxwyวnon ordonne la femme à s'assoir sur la natte (d) apprêtée pour la circonstance et la Toxwyəssi lui rasa les cheveux et pubis. Tous les objets réunit, sont accrochés à une souche d'arbre dans la forêt sacrée de la dite collectivité; les cheveux sont versés au pied du lègba familial appelé (houlègba) et on lui accrocha les perles qu'elle portait le jour de l'acte. Le Toxwyonon ramasse les cauris non percés ainsi que les colas, ata, ovi, apprêtés pour la circonstance, les jette par terre pour savoir la décision prise par les dieux. Le Təxwyวnon ramasse les jarres, les colas et tout ce qui a servir à la purification puis ils sortent (tous ceux qui ont assisté à la cérémonie de purification) de la forêt pour reprendre le même geste des cauris et des colas devant le chef de la collectivité et sa cour. La conformité des signes de la forêt avec ceux de la cour induit l'accord de grâce pour la femme infidèle. C'est alors que la tangninon et les houessi lui rappelèrent les prescriptions des lois et règles de la collectivité. Ainsi elle réapparait dans le cercle de la collectivité avec une âme pure exempte de péché. L'assise recommande maintenant à son mari de lui donner la popote pour la cuisine. Et le soir de la même journée, la purifiée prépare un met copieux de son choix à toute la famille. La nuit, elle va dormir désormais en paix avec son mari.

De l'analyse de cette description, il a été retenu que la culture « fon » d'abomey est une culture très attachée à ses savoirs endogènes. Ainsi elle se base sur ses savoirs pour s'assurer du maintien de stabilité conjugale en son sein. En effet, le rite de purification "afjlile" est une pratique sociale héritée par les descendants des ancêtres «fon». Elle a été instituée par leurs aïeux appelée (Tכxwy) dans le but d'accorder le pardon à toutes les femmes de la cours, "houessi", qui commettent l'adultère. C'est une pratique qui se déroule dans un cadre bien organisé, à un endroit précis, par le biais des objets bien déterminés et se fait suivant plusieurs étapes par différents acteurs initiés. Bien que le rituel de purification soit institué dans un but précis, il ne se déroule pas de la même manière dans les collectivités. De surcroit, dans les différentes cultures du Bénin. Par exemple chez les Wémènou d'Avagbodji (Bembè) à Aguégués, suite aux investigations menées sur le net, il a été révélé que le rituel de purification de la femme adultère se nomme "Ahanzin con yiyi" et c'est une pratique qui fait intervenir les "Tangninnon" (tantes âgées) qui sont chargées de la mettre en application. Ces rituels consistent à contraindre la femme à s'asseoir sur un tas de coque de noix de palme "Déyinkan" en lui faisant porter le "Ahanzin" (jarre sacrée) contenant de l'eau sacrée. Suite à l'invocation des Vodouns, l'inculpée est conviée à dire la vérité; ce qu'on appelle "Assouhihia". La manifestation de la vérité amène la femme à trembler et à faire trembler la jarre dans les mains jusqu'à s'évanouir: ce phénomène s'appelle "Ahanzin Hiho" et cela débouche sur la cérémonie de purification 
pour prouver que l'intéressée a été pardonnée, mais en cas de récidive c'est la mort qui survient. Par ailleurs, en cas de non aveu, la jarre pèse comme du béton dans les mains de l'inculpée et provoque une transpiration intense jusqu'à ce que cette dernière se fonde en larme. Mais malgré cela, deux nouvelles chances lui sont accordées afin de lui permettre de dire la vérité. Au cas où elle ne le ferait pas, elle serait répudiée. Et cette pratique a pour objectif d'accorder le pardon à toutes les femmes de la cours "Ahossi" qui commettent l'adultère dans cette ethnie.

Dans l'un ou l'autre des cas de purification, il y a des points de convergence lors des préparatifs pour le rituel proprement dit. Par exemple l'étape de "Assouhiha". Chez les «fon »d'Abomey, la "Tangninnon" pose à la femme adultère quelques questions dans le but de savoir le nombre d'hommes avec qui elle a couchée afin de voir dans quelle mesure s'y prendre. Les "Wémènou" d'Aguégués aussi passent par cette étape avant de purifier la femme adultère. La divergence se situe au niveau de la cérémonie proprement dite. Les «fon» procèdent par le lavage de la femme et le rasage de ses cheveux dans la forêt sacrée "Təxwy Zume" et les "Wémènou" procèdent par le "Ahanzin Hiho" sur un tas de coque de noix de palme "Déyinkan". Chaque culture se base sur ses propres savoirs pour purifier la femme adultère. En considérant ces deux cultures, on comprend que les cultures africaines sont très attachées à cette pratique, et rien de sérieux ne peut l'égalé ou la remplacé pour permettre à la femme de se maintenir encore au foyer après l'adultère. Elle véhicule le bon sens, la sagesse et l'humilité. Le rite ainsi dire est indispensable pour la communauté traditionnelle car il permet de se mettre en contact avec le monde invisible afin de mettre de l'ordre dans le monde visible. Dans ce sillage, il faut dire que toutes les traditions visent les mêmes objectifs en ce qui concerne cette pratique. "Le maintien de l'ordre social". Néanmoins, vu son caractère sacré, cette pratique se fait de diverses manières compte tenu des réalités de chaque culture. Alors bien qu'elle soit un point où se rassemblent les différentes cultures Béninoises, elle ne se pratique pas de la même manière.

\section{Perceptions sociales de l'adultère chez les "fon" d'Abomey Les expressions "AfJlile" et Afjogbé chez les "fon " d'Abomey}

A l'issue de cette recherche, il s'est avéré que quand on dit "AfJdogbé », cela ne renvoie pas à la traduction littérale qui désigne «la femme a amené ses pieds dans la brousse », mais plutôt qu' " elle a quitté son toit conjugal pour aller coucher avec un autre homme ». C'est alors une expression pour désigner l'adultère en milieu «fon ». De même, quand la femme commet l'adultère, c'est tout son être qui devient impur. Alors c'est son corps entier qui sera lavé et non seulement ses pieds. Alors, le lavage se fait de la tête aux pieds tout en prononçant des paroles incantatoires. Le 


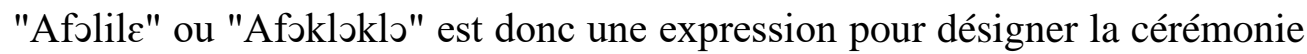
de purification de la femme adultère chez les « fon »d'Abomey.

\section{Le sort réservé au couple adultère}

L'adultère en tant que violation du devoir de fidélité entre époux, a été et reste considéré, dans de nombreuses sociétés comme répréhensible. Alors elle est proscrite dans ces milieux et sa transgression fait l'objet de malédiction en direction des conjoints. Deux principales versions sont dénombrées en la matière. La première version annonce que l'époux qui consent à l'adultère de sa conjointe subit un sort pouvant se matérialiser par une série de maladies qui échappent à la compétence de la médecine moderne dont le ballonnement du ventre et plus tard son décès. Pour les tenants de ce discours, l'époux qui tolère l'infidélité de sa femme provoque par la même occasion la «colère » des divinités protectrices de sa collectivité d'appartenance. Il se doit donc d'exprimer sa désapprobation en direction des sages et chefs de collectivité.

La deuxième version met en évidence une réaction des acteurs symboliques en direction de la femme ayant commis l'adultère. Les sages et femmes rencontrées racontent que si la femme garde le secret, elle suscite également la «colère » des divinités protectrices de la collectivité d'appartenance de son époux. Ainsi, l'évidence de «cauchemars » en cas de retour au domicile conjugale après l'acte est la manifestation de « la colère du monde des ancêtres » à l'encontre de la femme. Quelques sages attestent que certaines femmes perdent la vie dans certains ménages avec comme principale cause «l'adultère ». Elles peuvent contracter des maladies dont on ignore la provenance, soit ce sont ses enfants qui tombent malade ou la chute considérable de son commerce, de ses activités ou tout autre malheur. Plus rien ne marche pour elle et pour son foyer. Elle a pratiquement tous les malheurs du monde même la folie peut subvenir.

"Ça s'est passé chez moi et la femme ne faisait que tuer de poux dans son pagne, cela accompagné de rire sans motif et c'est la mort qui s'en est suivie ». [Entretien avec Nan G. A. B. 55ans, veuve et mère de quatre enfants.]

Cet extrait d'entretient montre à quel point l'adultère est un acte très difficile à intégrer dans nos sociétés traditionnelles car c'est la tradition même qui se charge du sort de la femme adultère et les corollaires qui y découlent, viennent du surnaturel. L'adultère n'est jamais une expérience facile à intégrer ou à dépasser. Alors, il est recommandé aux femmes de renoncer systématiquement, à leur mariage ou à la vie conjugale, une fois l'adultère commis. Un homme confiant la situation advenue à l'intérieur de sa collectivité d'appartenance : 
"Une femme l'a fait chez moi, et à son retour, elle est resté à l'écart de la maison et à demander à ses enfants de lui apporter quelques un de ses habits et est partie ». [Entretien avec B. P. $38 a n s$, entrepreneur, marié et père de 3enfants].

Ce passage nous permet de comprendre que le mieux pour la femme adultère c'est de ne même plus revenir à la maison après l'acte. Ainsi, elle sauve sa vie. Car si elle pénétrait la maison, elle serait sûrement confrontée à ces nombreux problèmes précités. Néanmoins, il y en a d'autres qui, juste après l'acte, cherchent des feuilles citées en haut (déssréssigèman, ahissihissi etc...) pour se purifier, elles-mêmes avant de rentrer à la maison. De là, leur forfait ne sera pas dévoilé et aucun malheur ne leur arrivera. Il faut noter que ces feuilles qu'elles cherchent pour s'auto purifier restent un secret que beaucoup de femmes n'ont pas encore découvertes. Alors attendons-nous au pire quand ce secret sera découvert par beaucoup de femmes.

\section{La place accordée à la femme après l'adultère}

L'homme est issu d'une famille, d'un clan et d'un lignage. L'individu isolé perd non seulement son statut de groupe, mais aussi ne possède plus toutes les facultés capables de s'associer aux autres. Lorsqu'une femme commet l'adultère, elle crée des handicaps vis-à-vis de sa propre personne. Elle perd sa place dans la société. Ainsi, son intégration au sein de la société devient difficile et suscite des démarches à mener. En tenant compte de son comportement, son époux devient réticent et partant de là, une source d'instabilité s'installe dans le couple et aussi dans la famille. Dans ce sillage, la femme adultère à Abomey ne vaut plus grande chose aux yeux de son mari et de sa belle-famille. Après l'adultère, la femme devient la risée de la société. Ainsi, elle subit une désintégration sociale. Partout où elle passe, elle est jugée sans aucune forme de procès par son entourage. On la traite de prostituée, de la pire des femmes ou elle subit des insultes de tous ordres. Au sein de son foyer, la méfiance s'installe, et elle n'a plus le droit de certaines décisions. Alors, pour éviter tous ces problèmes, certaines familles refusent catégoriquement de pardonner la femme si elle commet l'adultère. Ainsi, elle est répudié systématique de son foyer une fois l'acte commis. A cet effet, une enquêtée raconte ce qui suit :

«Chez nous les "AÏNON" quand la femme commet l'adultère, elle est systématiquement répudier de son foyer. Il n'y a pas demi-mesure. Il n'y a pas de négociation à faire. Chez nous, on ne connait pas ce qu'on appelle "Afolile". Si la femme commet l'adultère, elle part point tiret ». [Entretien avec A. G. 35ans, commerçante, mariée et mère de 3enfants] 
A travers ce passage, on comprend que dans certaines collectivités d'Abomey, il n'y a pas de mesure de purification de la femme adultère. Dans ce cas, la femme au foyer n'a pas intérêt à commettre l'adultère. Sinon, le divorce est le seul moyen de ne plus étoffer la situation d'adultère. C'est une manière de protéger sa vie. Il s'agit là d'une discrimination envers cette dernière. Puisque dans le même temps qu'on voit en infidélité (la polygamie) des hommes une norme, celle de la femme est considéré comme une déviance à corriger. Néanmoins, toujours à Abomey dans certaines familles comme chez les "ANANNOU", la femme adultère a droit à une seconde chance, même à une troisième c'est-à-dire que si la femme commet l'adultère deux fois, elle a droit à la cérémonie de purification pour sa réintégration dans son foyer et dans sa belle-famille si le mari décide de la garder bien sûr. Mais une troisième fois, elle est répudiée systématiquement. Notons que dans ces familles, quand une fois la cérémonie de purification est faite, nul n'a le droit de revenir en arrière, c'est-à-dire de revenir sur l'acte posé par la femme. Il est formellement interdit d'insulter la femme d'adultère ou d'avoir un regard méchant envers sa personne. Ainsi pour le mari que pour quiconque dans sa belle-famille. «Si jamais quelqu'un dans la famille remue le couteau dans la plaie c'est-à-dire insulte la femme de "agalèts" après sa purification, c'est les ancêtres mêmes qui se chargent de son sort. Car cette consigne a été donnée depuis la nuit des temps.» [Entretien avec dah Sagbadjou, 65ans, marié et père de 10 enfants.]

Cet entretien a permis de réaliser que la tradition, bien qu'elle a mis en place des moyens de régularisation sociale, c'est-à-dire des moyens de gérer l'infidélité au sein des couples, surtout celle de la femme, a aussi pensée au traitement que l'entourage peut avoir envers la femme adultère. Ainsi, elle interdit catégoriquement d'aborder ce sujet après la purification de la femme. Sinon, malheur à celui-là qui y revient. La femme qui insulte sa congénère adultère le sera à son tour. Il y a un adage que la femme adultère déjà purifiée tient souvent en face de certaines situations : "Enon zoun do noumè an. A zoun mion woui lo nan blo ».

C'est peut être une manière pour influencer afin d'éviter toute stigmatisation. Il est vrai qu'il est interdit de revenir sur l'adultère de la femme après la purification. Mais la communauté n'aura-t-elle pas un regard critique envers la femme ? Cela sera vraiment respecté par la communauté? Puisse que l'acte a été posé et le regard social va forcément changer. Et le fait d'interdire d'y revenir serait peut-être une excuse pour la femme de persévérer dans son forfait car elle se dira qu'elle a encore une chance de rester. Alors à Abomey, malgré qu'elles soient de la même ethnie « fon », les collectivités n'ont pas les mêmes prescriptions. Chez d'autres, on purifie la femme adultère 
afin de la garder dans son foyer. Par contre, chez d'autres c'est le renvoi systématique. Chaque collectivité à ses propres prescriptions et nul n'est audessus de celles-ci. C'est-à-dire que tu sois chef de collectivité ou quelle que soit ta responsabilité dans la collectivité, tu t'exécutes face aux règles de la maison sans protestation.

\section{L'utilité des rites de purification dans le processus de réintégration de la femme adultère}

L'Africain est d'une façon générale très croyant dans son essence. Pour être en contact permanent avec son créateur ou avec les divinités, il se met en condition pure à travers une cérémonie appelé "rite de purification". En se purifiant, l'homme se débarrasse de toute souillure. En effet, la purification peut concerner un seul individu comme toute une communauté. Elle n'est pas propre à une société. Chaque société a ses méthodes de purification. Et elles se pratiquent sous différentes formes et varient suivant les circonstances. La purification est un acte symbolique. Cet acte a pour objectif de conditionner mentalement l'être humain à être plus réceptif. Elle sert également à mettre en confiance celui qui la pratique. Il se sent psychologiquement protégé par ce rituel contre les "forces du mal". Elle est destinée soit à attirer la protection des dieux sur des individus ou sur toute la collectivité, soit à chasser loin d'eux "les forces du mal", soit encore à les remettre en harmonie avec leur environnement. Ainsi, dans le contexte socio-culturel des «fon», quand la femme commet l'adultère, elle est considérée comme une criminelle aux yeux de tous, car l'adultère est un acte proscrit par la tradition africaine et ce n'est qu'après avoir été purifiée au cours d'une cérémonie spéciale qu'elle redevienne telle qu'elle était avant l'acte aux yeux des ancêtres et aux yeux de toute la famille. A travers ces rites, on implore le pardon des ancêtres afin d'atténuer les conséquences de son acte pour lui donner une nouvelle chance. L'attitude de respect qu'institue le rite permet de renforcer la cohésion familiale et clanique, de canaliser des émotions communes. Le rite permet donc non seulement de purifier la femme mais aussi d'amener de l'harmonie dans la famille. Le rite permet aussi de renforcer les rôles sociaux de certains individus, de réaffirmer les règles et les normes, il structure et dynamise les comportements, atténue les tensions sociales. Il renforce la motivation et la mobilisation autour de buts communs. Il rassemble périodiquement une communauté dans une union symbolique, il a pour conséquence une perpétuation et un renouvellement des croyances. Les purifications servent à nettoyer non seulement le corps, mais principalement l'esprit.

\section{Discussion}

L'analyse des résultats montre que dans la tradition «fon», la consultation du "fâ" est une démarche habituelle pratiquée par toute la 
population, ce que M. Dixsaut (2002, p. 38) présente comme une manière d'accéder à la connaissance de soi. En d'autres termes, « le "fâ" est le pont qui unit le temps des origines au temps profane. A travers ses mythes et légendes, le "fâ" fournit à l'homme des exemplaires pour une existence récente dans un monde désaxé » (M. Kakpo, 2009, p. 52). Ainsi à travers les révélations du "fâ", on arrive à comprendre ce qui se passe dans le monde invisible.

Dans cette même logique C. Rivière (1997, p. 160) explique cette pratique comme « des relations entre les hommes et les dieux qui se font dans un cadre rituel, ensemble de conduites et d'actes répétitifs et codifiés, souvent solennels, d'ordre verbal, gestuel et postural, à forte charge symbolique, fondés sur la croyance en la force agissante d'êtres ou de puissances sacrées, avec lesquelles l'homme tente de communiquer en vue d'obtenir un effet déterminé ». Toujours selon cet auteur « le rituel est la machine à produire et à reproduire du social par excellence ». Néanmoins, vu son caractère sacré, cette pratique se fait de diverses manières compte tenu des réalités de chaque culture. Alors bien qu'elle soit un point où se rassemblent les différentes cultures Béninoises, elle ne se pratique pas de la même manière.

Les enquêtes de terrain font également découvrir que l'adultère est un acte très difficile à intégrer dans les sociétés traditionnelles car c'est la tradition même qui se charge du sort de la femme adultère et les corollaires qui y découlent, viennent du surnaturel. Et comme l'affirmait A. Houel (2007, p. 22), « une infidélité ou une rupture produit d'intolérables douleurs, voire de très profonds dégâts ». Lorsqu'une femme commet l'adultère, elle crée des handicaps vis-à-vis de sa propre personne. Elle perd sa place dans la société. Comme l'a expliqué E. Charvier-Berman, (1989, p.49) : "En commettant un acte adultère, la femme refuse le rôle d'épouse fidèle. Elle refuse ainsi le contrat social préétabli, et introduit une rupture dans l'ordre social ». Ainsi, son intégration au sein de la société devient difficile et suscite des démarches à mener. Dès lors la femme adultère subit une stigmatisation terrible. Car comme l'a dit L. Mucchielli (2002, p. 52), la cause du crime, c'est le criminel lui-même. Contrairement à cette opposition à la réintégration L. Bussière (2009, p. 45) aborde l'importance de la purification des femmes adultères qui les plongent dans une communion ou une rencontre avec le sacré, qui joue un rôle dissuasif. Cet acte a pour objectif de conditionner mentalement l'être humain à être plus réceptif. D'ailleurs, symboliser, c'est représenté le réel et établir un rapport de signification entre les choses (Gerstlé, 1997, p. 89).

A contrario, O.D. Dima (2010, p. 260) considère l'adultère comme une nécessité physique, émotionnelle, spirituelle et sociale. Il indique dans son analyse que l'adultère est un substitut nécessaire à certains aspects importants dans la vie, souvent absents dans les mariages. Ces aspects manquants peuvent être la passion, le désir et le plaisir. De cette façon, la conséquence de son adultère est pareille à ce dont parle H. Neuschäfer (1989, p. 39), qui dans son 
analyse montre comment l'adultère féminin devient une sorte de libération qui contribue à rendre la femme plus indépendante. Mais cette vie de débauche n'est pas sans conséquences sur sa santé puisqu'elle est exposée à des maladies sexuellement transmissibles telles que le VIH Sida. Et aussi sur sa dignité, puisse qu'elle devient la risée de la société et le regard à son endroit change. A cet effet, E. Joachimsson (2015, p. 27) dépeint la manière dont le mariage forcé, sans amour, accentue les différences entre les deux êtres, ce qui résulte en une incompréhension totale dans le couple. E. Joachimsson (2015, p. 27) trouve que les raisons de tromper sont le désaccord entre les époux, le manque d'amour, la volonté de changer la vie monotone, une façon de se venger contre l'indifférence du mari. Alors, il est compris à travers ces propos qu'un mariage fondé sans amour et sans consentement débouche souvent sur des conflits entre les deux conjoints, source d'adultère.

\section{Conclusion}

Les rites de purification interviennent pour restaurer la cohésion familiale lorsqu'une femme commet le péché social, culturel et cultuel : l'adultère. A la question de savoir l'importance des rites et s'il faut toujours recourir à ces derniers, les opinions recueillies sont variables selon l'adoption de la culture de la famille. Nous somme alors en présence de deux différentes visions, celle dans laquelle les rites de purification (afjlile) sont accordés à la femme adultère dans le but d'implorer la clémence des ancêtres afin de la conserver dans son foyer. Cette première vision est celle qui interdit catégoriquement la marginalisation ou la stigmatisation de la femme adultère une fois que les cérémonies sont faites. La deuxième est celle dans laquelle quand la femme commet l'adultère, elle est systématiquement répudier de son foyer. Cette perception de l'adultère est basée sur le fait qu'une femme adultère est une criminelle qui n'a plus sa place au sein de la société. Les rites de purification conçus dans un itinéraire de réintégrer la femme adultère afin de lui redonner sa place, constituent une stratégie développée par la culture «fon » d'Abomey pour assurer l'équilibre du couple et la cohésion familiale. Mais bien que ces rites soient disponibles et constituent un moyen pour le contrôle social, et malgré que bon nombres de familles dans nos sociétés traditionnelles s'attachent à ces rites pour conscientiser la femme, force est de constater que rien n'empêche la femme à commettre ce forfait une fois que l'occasion se présente à elle. Ainsi le phénomène persiste.

\section{References:}

1. Bussière Luc, 2009, Evolution des rites funéraires et du rapport à la mort dans la perspective des sciences humaines et sociale, Ecole des Etudes Supérieures, Université Laurentienne Sudbury, Ontario.

2. Cazeneuve Jean, 1971, Sociologie du rite, Paris : PUF, p.106. 
3. Charvier-Berman Evelyne, 1989, « Maupassant nouvelliste : personnage féminin et adultère ». Paroles gelées $\mathrm{n}^{\circ} 7$ (1), pp.43-50.

4. De Singly, François, 1996, Le Soi, le Couple et la Famille, Paris, Nathan.

5. Dima Oana-Cristina, 2010, «L'adultere chez Maupassant ». In : The Proceedings of the International Conference "Communication, Context, Interdisciplinarity". Section: Language and Discourse. $\mathrm{n}{ }^{\circ} 1$ 2010, pp.258-263.

6. Dixsaut Monique, 2002, La connaissance de soi: études sur le traité 49 de Plotin. Vrin, 2002.

7. Flaubert Gustave, 2006, Madame Bovary, Paris : Librairie Générale Française.

8. Gerstlé Jacques, 1997, La persuasion de l'actualité télévisée. Politix. Revue des sciences sociales du politique, vol. 10, no 37, p. 81-96.

9. Houel Annik, 2007, Les contradictions de l'adultère féminin. Souffrance et plaisir dans la vie amoureuse, Le Journal des psychologues, Vol. 6, $\mathrm{n}^{\circ}$ 249, pp. 20-24.

10. Joachimsson Elin, 2015, Le thème de l'adultère dans Une vie et Belami de Guy de Maupassant.

11. Kakpo Mahougnon, 2009, Introduction à une poétique du Fa, Diaspora, Cotonou Bénin, p.192.

12. Leleu Gérard, 2004, La fidélité et le couple, Paris : Flammarion.

13. Mucchielli Laurent et Robert Philippe, 2002, Crime et sécurité : un état des savoirs, Paris, La Découverte.

14. Naouri Aldo, 2006, Adultères, Éditions Odile Jacob.

15. Neuschäfer Hans, 1986, «Le déclin du patriarcat, Adultère et divorce dans le roman-feuilleton de 1884 », Littérature populaire, $n{ }^{\circ} 53$, pp. 37-48.

16. Paquette Danielle, 2004, «La relation père-enfant et l'ouverture au monde ». Enfance, vol. 56, no 2, p. 205-225.

17. Rivière Claude, 1998, Socio-anthropologie des religions, Archives de sciences sociales des religions, Vol. 104, $\mathrm{N}^{\circ} 1$, pp.160-161

18. Thomas Louis-Vincent et Lumeau René., 1986, La terre africaine et ses religions, Paris : 1'Harmattan, p.203. 